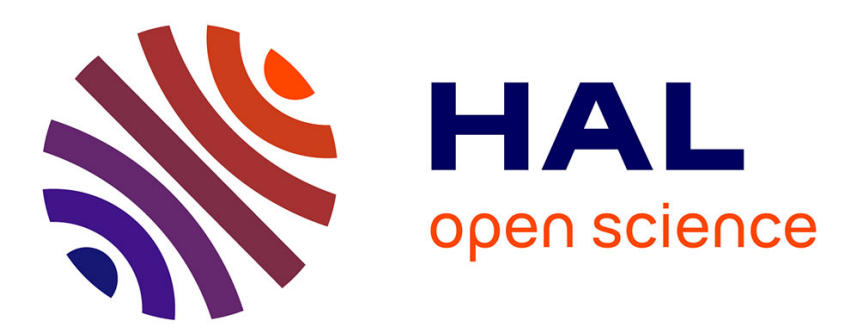

\title{
Aux origines de la Catalogne: le mythe fondateur de la Maison de Barcelone dans l'historiographie du XIIe siècle
}

Martin Aurell

\section{- To cite this version:}

Martin Aurell. Aux origines de la Catalogne: le mythe fondateur de la Maison de Barcelone dans l'historiographie du XIIe siècle. Comptes-rendus des séances de l'Académie des inscriptions et belleslettres, 1998, 142 (1), pp.7-18. 10.3406/crai.1998.15826 . halshs-01333546

\section{HAL Id: halshs-01333546 \\ https://shs.hal.science/halshs-01333546}

Submitted on 17 Jun 2016

HAL is a multi-disciplinary open access archive for the deposit and dissemination of scientific research documents, whether they are published or not. The documents may come from teaching and research institutions in France or abroad, or from public or private research centers.
L'archive ouverte pluridisciplinaire HAL, est destinée au dépôt et à la diffusion de documents scientifiques de niveau recherche, publiés ou non, émanant des établissements d'enseignement et de recherche français ou étrangers, des laboratoires publics ou privés. 


\section{Aux origines de la Catalogne : le mythe fondateur de la Maison de} Barcelone dans l'historiographie du XIle siècle

Monsieur Martin Aurell

Citer ce document / Cite this document :

Aurell Martin. Aux origines de la Catalogne : le mythe fondateur de la Maison de Barcelone dans I'historiographie du XIle siècle. In: Comptes rendus des séances de l'Académie des Inscriptions et Belles-Lettres, 142 e année, N. 1, 1998. pp. 7-18; doi : 10.3406/crai.1998.15826

http://www.persee.fr/doc/crai_0065-0536_1998_num_142_1_15826

Document généré le 05/06/2016 


\title{
COMMUNICATION
}

\author{
ALX ORIGINES DE LA CATALOGNE: \\ LE MYTHE FONDATELR DE IAA MAISON DE BARCELONE* \\ DANS IUHISTORIOGRAPHIE DU XI广 SIECLE, PAR M. MARTIN AURELL
}

Les anciens racontent qu'ily avait, jadis, un chevalier nommé Guifred, originaire du village de Rià, situé dans le territoire de Conflent, sur les rives du Tet, près du monastère de Sant Miquel de Cuixà. Ce chevalier, très grand en richesses, en armes et en conseil, reçut le comté de Barcelone des mains du roi des Francs en raison de sa probité. Un jour, il se rendit à Narbonne avec son fils Guifred, qu'on surnommait le Velu, afin d'avoir un entretien avec les envoyés du roi. Au cours d'une rixe, il tua, de son glaive, un Franc qui lui avait tiré la barbe. Il fut pris aussitôt et, tandis qu'on le conduisait en France auprès du roi, une nouvelle rixe éclata, parce qu'il voulait se venger de sa captivité : on raconte qu'il fut tué par son escorte, près du Puy-Notre-Dame. Son fils Guifred, qui était emmené avec lui, fut livré au roi des Francs, auquel on exposa ce qui était advenu à son père pendant le voyage. Le roi en fut très attristé : il critiqua leur action et leur annonça que, dars l'avenir, le roi des Francs pourrait perdre, de ce fait, le domaine de Guifred.

Ayant accueilli l'enfant, il le confia, d'après ce que l'on rapporte, au comte de Flandre pour qu'il l'éduquât avec beaucoup de soin. Devenu adolescent, Guifred rendit enceinte la fille de ce comte, sans que personne ne s'en aperçût, sauf, avec perspicacité, la mère de la fille, qui garda le secret, beaucoup plus par pudeur que parce qu'elle y consentît. Ne sachant à quel mari la donner dorénavant, car sa fille serait couverte d'opprobre si la nouvelle se diffusait, elle trouva enfin cette solution : elle fit venir le jeune homme et lui fit jurer que si, par la volonté de Dieu, il parvenait à recouvrer le domaine paternel, c'est-à-dire le comté de Barcelone, il s'unirait à sa fille en mariage.

Elle le vêtit, ensuite, pauvrement, en habit de pèlerin, et l'envoya, accompagné d'une vieille femme, auprès de sa mère qui vivait encore, veuve, dans le territoire de Barcelone. Celle-ci le reconnut à la pilosité qu'il avait dans des endroits peu habituels du corps de l'homme et qui lui avait valu le sobriquet de Velu. Elle convoqua les magnats et les barons de toute sa terre, qui avaient connu son père et qui lui étaient restés fidèles: elle leur présenta son fils en secret. Ces magnats et nobles, à la pensée de la fraude et de l'opprobre qui avaient causé l'assassinat de son père et sa

* Les tableaux généalogiques de la maison de Barcelone, pour les X"-xul" siècles, figurent en fin de communication, p. 17 sq. (́abl. 1 et 2 ;. 
propre exhérédation, le prirent pour seigneur et lui jurèrent fidélité, s'engageant à le servir. Ils fixèrent ensuite une date et se présentèrent, ensemble avec le jeune, à l'endroit où ils avaient décidé que Salomon, gaulois par sa nation et alors comte de Barcelone, mourrait: là, du consentement général, le jeune sortit son épée et le tua, devant tous, de ses propres mains. Il obtint ainsi, à vie, pour lui tout seul, son comté, sis entre Narbonne et l'Espagne. Enfin, ayant envoyé des messagers en Gaule, il épousa, comme promis, la fille du comte de Flandre et alla la prendre au lieu et au jour convenus. Il gagna, avec le conseil et l'aide des amis de la jeune fille, la grâce et l'amitié du roi: après avoir reçu son domaine de ses mains, il resta longtemps à sa cour.

Alors qu'il y demeurait encore, la nouvelle arriva que les sarrasins étaient entrés dans sa patrie et l'avaient, presque toute, parcourue et occupée. Il en fit part au roi, lui demandant de l'aide pour les expulser. Mais le roi, empêché par d'autres affaires, ne put lui prêter secours. Il accéda cependant à l'une de ses requêtes : si Guifred parvenait, de son côté et avec les siens, à buter les Agaréens hors de ses terres, le domaine de Barcelone passerait perpétuellement sous son autorité et sous celle de sa dynastie; avant lui, en effet, le roi des Francs n'avait jamais cédé de comtés par succession héréditaire, mais il les accordait, à son gré, à la personne qu'il voulait pour un temps déterminé. Guifred réunit, de part et d'autre, de nombreux barons gaulois et expulsa les Agaréens de toutes ses terres jusqu'à la frontière de Lérida. Il posséda en propre tout ce domaine, qu'il avait récupéré avec tant de vaillance.

Et c'est ainsi que le domaine de Barcelone passa du pouvoir royal aux mains de nos comtes !

Tel est le commencement des Gesta comitum Barchinonensium, le premier texte historique destiné à exalter la dynastie barcelonaise : il relate, sans préambule aucun, l'aventure de Guifred le Velu, ancêtre fondateur de la lignée. Ce récit ouvre, de façon significative, les Gestes qui - leur titre ne devrait pas nous induire en erreur - ne sont rien d'autre qu'une généalogie ${ }^{2}$, dressant, à l'aide répétitive des verbes genuit et successit, la liste des descendants du premier héros de la maison et consignant, au passage, quelques allusions furtives à leurs hauts faits d'armes'. C'est entre 1162 et 1184 que leur version primitive, dont nous avons traduit le passage ci-dessus, a été rédigée : Alfons I'v vient d'accéder à la royauté, d'unir 3-5.

1. Gesta Comitum Barchinonensium, L. Barrau Dihigo et J. Massó éd., Barcelone, 1925, p.

2. Ils cadrent parfaitement avec la définition proposée par G. Duby, Hommes et structures du Moyen Âge, Paris, 1973, p. 287-298.

3. Th. N. Bisson, UUnheroed Pasts : History and Commemoration in South Frankland before the Albigensian Crusades», Speculum, 1990, p. 299 ; J. P. Rubiés, J. M. Salrach, antorn de la mentalitat i la ideologia del bloc de poder feudal a través de la historiografia medieval fins a les quatre grans cròniques", dans La formació i l'expansió del feudalisme català, Gérone, 1985, p. 479. 
en sa personne la Catalogne et l'Aragon, de récupérer la Provence, d'annexer le Roussillon et de parfaire la reconquête de la Nouvelle Estrémadure aragonaise. La célébration de son pouvoir souverain, de son expansion et de ses succès militaires s'impose.

Les moines de Ripoll, gardiens de la nécropole comtale, écrivaient, de longue date, des épitaphes en l'honneur de ses ancêtres ${ }^{4}$; inscriptions lapidaires, rouleaux des morts et oraisons funèbres conservaient ces élégies versifiées. L'un de ces parchemins relatant les hauts faits de la maison princière a été même retrouvé dans le tombeau de Ramon Bérenguer IV (1130-1162), signe irréfutable du lien qu'entretiennent alors l'historiographie et la mort. Année après année, les bénédictins remémoraient, en effet, les défunts de la famille comtale dans l'office monastique : des obituaires rappelaient la date anniversaire de leur trépas. A partir de ces nécrologes, simples calendriers liturgiques annotés, et à partir des listes des rois de France, indispensables pour dater les actes, ils avaient commencé, à la fin du $\mathrm{X}^{\mathrm{e}}$ siècle, à écrire inconsciemment de l'histoire ${ }^{5}$. Leurs annales et chronicons exposaient brièvement les principaux événements ${ }^{6}$. Cette première expérience, unie à la constitution d'une des plus riches bibliothèques de l'Occident médiéval, prédisposait, à la fin du XII ${ }^{\mathrm{e}}$ siècle, les intellectuels du scriptorium de Ripoll à rédiger un texte historiographique apte à justifier la domination barcelonaise, celui dont nous venons de lire le début. $\mathrm{Du} \mathrm{XIII}^{e}$ au $\mathrm{XV}^{e}$ siècle, cet écrit primitif devient le canevas qui, dûment enrichi, sert aux scribes des comtes pour raconter l'histoire de leur lignée. Il ne reste, cependant, qu'une seule version de ce récit dans son état original, celle qui fut copiée au moment de sa composition dans un registre en parchemin de Ripoll, qui, acquis au XVII siècle par Étienne Baluze, est aujourd'hui conservé à la Bibliothèque nationale'. En 1925, cette histoire première a fait, avec les autres versions des Gesta comitum Barchinonesium, l'objet d'une édition remarquable par Louis Barrau Dihigo et Jaume Massó.

Au Moyen Âge, les moines de Ripoll entretenaient des relations plus que suivies avec le monastère tout proche de Sant Miquel de

4. Sur le lien entre épitaphe et généalogie, cf. G. Duby, op. cit. (n. 2), p. 294.

5. Peut-être les comtes avaient-ils encouragé l'essor de l'historiographie à Ripoll, monastère sûr, protégé, au nord de leur domaine, des raids musulmans, à la suite des destructions des archives barcelonaises par al-Mansur en 985 , comme le propose M. Coll, «La historiografia de Catalunya en el periode primitius, Estudis romànics, 1951-1952, p. 146. Cf. M. Zimmermann, \&La prise de Barcelone par al-Mansûr et la naissance de l'historiographie catalane., Annales de Bretagne et des pays de l'Ouest, 1980, p. 191-218.

6. M. Coll, art. cit. (n. 5), p. 155 sqq.; Th. N. Bisson, art. cit. (n. 3), p. 287-289.

7. Manuscrit latin 5132, fol. 23v-25v. 
Cuixà : au début du XI' siècle, l'abbatiat double d'Oliba de Cerdagne, évêque de Vic, témoigne de cette sorte de jumelage. C'est dans le cloître de cette dernière maison que la légende de Guifred le Velu introduisant les Gesta avait été réélaborée à partir d'un conte populaire : les éléments topographiques du début du récit permettent cette localisation. Tout récemment, Miquel Coll a même avancé le nom de Gregori († 1146), abbé de Cuixà, comme celui de son auteur, qui aurait transmis cette tradition ancienne aux scribes de Ripoll : en 1139, ce prélat fut choisi en tant qu'archevêque de Tarragone, la plus haute dignité ecclésiastique catalane, par Ramon Berenguer IV, peut-être en récompense pour les services que son érudition avait prêtés à la couronne $e^{8}$. Cette hypothèse, désignant l'intermédiaire culturel entre le récit folklorique et sa fixation savante, est séduisante : le rédacteur ou, du moins, l'inspirateur du mythe des origines de la dynastie avait ses entrées à la cour. Il avait envisagé sa tâche d'historien avec plus de ferveur patriotique que de rigueur scientifique.

$\mathrm{Sa}$ légende contenait certes quelques éléments véridiques, empruntés aux événements du IX ${ }^{e}$ siècle : en 843 , le père de Guifred le Velu, Sunifred I ${ }^{\text {er }}$ que les Gesta appellent, par mégarde, Guifred, avait reçu Prada, en Conflent, de Charles le Chauve ; ce Goth eut maille à partir avec Salomon, un Franc peut-être, qui le remplaça, en 848, à la tête des comtés d'Urgell et de Cerdagne et qui tenta, en 868 , de spolier Cuixà des donations des parents de Sunifred $I^{\text {er }}$; en 870 , Guifred le Velu lui succédait en tant que comte de Cerdagne et expulsait les musulmans du comté de Vic ${ }^{9}$. L'historicité des principaux protagonistes de notre récit ne doit cependant pas faire oublier que son auteur en sait davantage sur le $\mathrm{XII}^{\mathrm{e}}$ siècle, qu'il vit intensément, que sur un vague $\mathrm{IX}^{\mathrm{C}}$ siècle, dont le souvenir collectif et les rares renseignements glanés dans les archives monastiques lui transmettent une bien pâle image.

Le texte respire un évident chauvinisme anti-français : les assassins de Guifred sont des émissaires francs; Salomon, l'intrus tué au cours d'un duel judiciaire, est de nation gauloise; le roi de France est incapable de combattre les musulmans. Au moment de

8. M. Coll, Guifré el Pelós en la historiografia i en la llegenda, Barcelone, 1990, p. 20.

9. Cf. P. Ponsich, Le nôle de Saint-Michel-de-Cuxa dans la formation de l'historiographie catalane et l'historicité de la légende de Wilfred le Velu», Études roussillonnaises, 19541955 , p. 156-159. J. M. Salrach (El procés de formació nacional de Catalunya (segles VIII-IX), Barcelone, 1978, t. II, p. 38 sqq. et $92 . s q$.), tout en reconnaissant une certaine historicité à notre légende, nie, à la suite de R. d'Abadal, les origines franques de Salomon et son caractère d'intrus. Le procès par lequel, en 862 , Salomon déposséda un fidèle de Sunifred I"r des biens que celui-ci lui avait cédés ainsi que l'action qu'il intenta contre Cuixà, en 868 , montrent pourtant qu'il y a eu une opposition politique, qui découle, peut-être, de la différence ethnique avancée par les Gesta. 
sa rédaction, les rapports avec la royauté ne sont précisément pas au beau fixe. Ramon Berenguer IV vient de nouer une solide alliance avec Henri II Plantagenêt, l'ennemi invétéré du roi de France : il le nomme, sur son lit de mort, tuteur de ses enfants. Son fils, Alfons $I^{\text {er }}$ combat, sa vie durant, Raimon V de Toulouse, le gendre du Capétien. Une certaine aversion pour le roi pourrait s'expliquer par les choix politiques des Barcelonais, favorables aux Plantagenĉt. Mais ni la diplomatie ni la guerre ne rendent compte, à elles seules, de la francophobie des Gesta: ni Louis VI (11081137) ni Louis VII (1137-1180) n'ont encore une action systématique dans le Midi et, s'ils parviennent à agir au-delà de l'île-deFrance, leur action ne se concrétise guère qu'en Flandre, en Normandie, voire en Auvergne. Au XIr siècle, les Capétiens sont encore trop lointains pour inquiéter outre mesure les Catalans dans leur politique méridionale.

Notre légende apparaît, en fait, comme une tentative de légitimer l'indépendance des comtés catalans vis-à-vis de la couronne : le roi lui-même prophétise que le meurtre du Puy lui fera perdre ces territoires et promet à Guifred qu'il pourra fonder, en dépit de la tendance centralisatrice de sa politique, sa propre lignée comtale s'il parvient à refouler les sarrasins ${ }^{10}$. La moralité de l'histoire est nettement exprimée dans sa phrase conclusive : «Et c'est ainsi que le domaine de Barcelone passa du pouvoir royal aux mains de nos comtes! » Son auteur apporte une justification théorique à l'autonomie que ces princes s'étaient donnée, dans la pratique, depuis trois siècles.

Une décision, prise à l'époque où cette anecdote était couchée sur parchemin, montre le souhait du comte de Barcelone, devenu roi, de se dégager de toute autorité supérieure. En 1180, au cours du synode de Tarragone, Alfons Ir enjoignit aux scribes de sa principauté d'abandonner l'habitude archaïsante de dater leurs actes d'après les années de règne des rois de France : son ordre fut largement respecté". Cette modification, à forte teneur symbolique, de la diplomatique catalane traduit une volonté manifeste d'accéder à la souveraineté ; elle participe du même esprit qui anime la légende de Guifred le Velu. En tant que monarque, le comte de Barcelone se place désormais au sommet de la hiérarchie des pouvoirs.

10. Pour comprendre la démission du roi de France dans la lutte contre l'Islam et la justification du pouvoir indépendant des comtes de Barcelone, il faut se placer dans le contexte du sac d'al-Mansur de 985 et de l'avènement d'Hugues Capet, à la légitimité douteuse, en 987 : M. Zimmermann, art. cit. (n. 5).

11. M. Zimmermann, a La datation des documents catalans du IX au XII siècle : un iti. néraire politique», Annales du Midi, 1981, p. 345-375. 
La xénophobie anti-franque des auteurs des Gesta ne répond donc pas à une quelconque présence menaçante des troupes royales en Occitanie, qui aurait pu entraver l'expansion territoriale des Catalans. Leur rejet est plutôt constructif : la conscience nationale s'affirme, à l'époque, autour de la dynastie barcelonaise; pour s'imposer, elle trouve son pôle répulsif dans une altérité capétienne, fortement récusée. Tout acte d'identité, aussi positif ou défensif qu'il puisse paraître, comporte une dimension négative, offensive ou agressive : le groupe se définit par opposition à autrui. Le sentiment anti-capétien n'est ici qu'un comparse dans la commémoration de la montée du pouvoir dynastique barcelonais.

Le mariage fondateur entre Guifred le Velu et la fille du comte de Flandre est l'élément central de notre récit. L'assassinat du père a dépouillé son protagoniste de ses biens. C'est pourquoi il est élevé dans la demeure du comte de Flandre, devenu son protecteur et seigneur, dont il laisse la fille enceinte. Ce sera encore l'errance, le retour au pays dans les pauvres habits du pèlerin, pour reprendre le patrimoine ancestral à la suite d'un duel judiciaire dont il est le courageux champion. Son exploit lui vaut la main de son amante. Réconcilié avec le roi et vainqueur des sarrasins, Guifred peut, enfin, fonder une dynastie comtale indépendante. Son union s'avère féconde: elle est la souche des nombreuses ramifications généalogiques dont les moines de Ripoll dressent, aussitôt, la liste dans les Gesta.

L'ancêtre des comtes de Barcelone est un aventurier, un jeune en quête de fortune et de renommée dans un inlassable voyage. Dans un article suggestif, E. Bournazel a dressé la liste de ces héros mythiques, souches des grandes lignées d'Occident qui, partis de rien, jettent, par leurs faits d'armes, les bases d'une grande famille ${ }^{12}$. Leurs exemples foisonnent dans la littérature généalogique de la fin du XII ${ }^{e}$ siècle: Gero, chef normand, aïeul des Blois ; scandinaves aussi, Achard, souche des comtes de Barsur-Aube, et Raoul Barbeta, créateur de la famille de Roucy ; d'extraction modeste, Archambaud et son inséparable compagnon Gouffier se trouvent à l'origine des vicomtés de Comborn et de Lastours ; ils rappellent Tertulle le Forestier, ancêtre premier des comtes d'Anjou. Les Robertiens, eux-mêmes, sont, sous la plume d'Aimon de Fleury (965-1008), les descendants de Widukind, le rebelle saxon maté par Charlcmagne : l'élection d'Eudes au trône de France ne leur apparaît que comme une revanche posthume, préparant le triomphe d'Hugues Capet. Pourvu qu'il soit chevale-

12. .Mémoire et parentés, dans La France de l'an mil, R. Delort éd., Paris, 1990, p. 114-124. 
resque, le modèle de l'homme nouveau, bâtissant à la force du poignet une maison nobiliaire, ne dérange nullement les vieilles familles princières.

Dans les généalogies qu'ils commandent au cours des années 1150-1200, les princes territoriaux font sourdre un aventurier fondateur de la mémoire originelle de leur famille. Cet ancêtre, « glorieux repère de l'élévation familiale , ne leur appartient pas en propre : ils l'ont emprunté à l'idéologie de l'aristocratie châtelaine nouvelle, dont le succès date, à peine, de l'effondrement de l'Empire carolingien. Le mythe des origines qu'adoptent les comtes est le même que celui des nobles qu'ils essayent de ramener sous leur autorité. Son modèle est lignager.

Le choix de la Flandre comme théâtre des amours de Guifred le Velu mérite d'être relevé ${ }^{13}$. En 1194, Lambert d'Ardres y finissait la généalogie des comtes de Guines, dont il était le chapelain. Or, il plaçait, à l'origine de la maison de ses mécènes, Sigfried, un aventurier scandinave, bâtisseur de la forteresse qui, au cour de son patrimoine, devait faire sa puissance : cet étranger séduisit la fille du comte de Flandre; il en eut un fils illégitime, adopté par son beau-frère, l'oncle maternel de l'enfant, qui l'adouba ${ }^{14}$. Dans une chanson de geste en langue d'oïl, Otger le Danois, prisonnier du châtelain de Saint-Omer, engendre Baudouin de la fille de son geôlier: Carlot, fils de Charlemagne, tue cet enfant, qu'Otger venge dans une lutte impitoyable contre les Francs ${ }^{15}$. Le thème des guerriers scandinaves, séducteurs de la fille du comte de Flandre et ennemis du roi de France, se diffuse oralement.

Ces épisodes étaient devenus un lieu commun de la littérature flamande à la suite d'un mariage réel. Baudouin Ier Bras-de-Fer $(\uparrow 878)$, comte de Flandre et fondateur de sa lignée, n'avait-il pas épousé Judith, fille de Charles le Chauve, contre la volonté de ce roi ? Les Annales de Saint-Bertin, dont les monastères pyrénéens conservaient des manuscrits, rapportaient cette histoire, prouvant que la maison de Flandre descendait, par les femmes, des Carolingiens ${ }^{16}$. Le sang de la lignée impériale devait couler, de même, dans les veines des comtes de Barcelone, comme dans celles des

13. Les historiens catalans insistent sur l'hostilité envers les Français dont fait preuve Thierry d'Alsace (1128-1168), comte de Flandre et paladin de l'indépendance de sa principauté, dans sa lutte contre Louis VI : M. Coll, op. cit. (n. 8), p. 19. Cet épisode pouvait, tout au plus, accroître la réputation de la maison de Flandre en Catalogne. Mais il n'explique nullement, à lui seul, que les comtes de Barcelone aient choisi une femme de cette maison comme première ancêtre. L'aspect généalogique - les origines carolingiennes des Flamands - paraît bien plus décisif.

14. G. Duby, op. cit. (n. 2), p. 280.

15. M. Coll, op. cit. (n. 8), p. 19 sq.

16. Th. N. Bisson, Medieval France and her Pyrinean Neighbours, Londres, 1989, p. 138. 
comtes de Guines, puisque leurs ancêtres respectifs avaient séduit une fille du Flamand: ces deux héros originels suivaient, de la sorte, l'exemple de Baudouin $I^{\text {er }}$, auteur du rapt de Judith, dont ils épousaient la descendante.

Le modèle de l'ancêtre fondateur apparenté à l'Empereur par un mariage hypergamique semble, cette fois-ci, bien plus royal que lignager: les Capétiens l'adoptent à la fin du XII' siècle. A l'occasion de l'intronisation de Louis VIII (1223-1226), né de l'union entre Philippe Auguste (1180-1223) et Isabelle de Hainaut, qui avait Charles de Lorraine pour aieul, les intellectuels de la cour insistent sur le sang carolingien qui les irrigue désormais. Le thème du « Retour du royaume à la race de Charlemagne», Reditus regni ad stirpem Karoli Magni, est exploité, au XIII siècle, par l'historiographie de Saint-Denis, la nécropole monastique des rois de France $^{17}$. Le mariage flamand de Guifred le Velu donnait un lustre supplémentaire à la royauté du comte de Barcelone.

Dans la légende des Gesta, la femme ne joue pas simplement un rôle passif: son image va bien au-delà de la belle proie capturée par un aventurier qui se donne, à travers elle, une postérité de sang royal. La fille du comte de Flandre est une bien falote actrice en comparaison des autres dames qui traversent le théâtre de notre histoire. Par contraste, sa mère marque puissamment de sa griffe le cours des événements. Elle découvre, d'abord, la grossesse, dont elle garde le plus grand secret pour ne pas couvrir d'opprobre ni sa fille déflorée ni sa famille déshonorée : le gynécée apparaît à l'écart des regards indiscrets des hommes, qui ne savent pas en percer tous les mystères. Elle dicte, ensuite, à son futur gendre la conduite à suivre, le déguisant, comme il convient à ses méthodes cachottières, pour faciliter sa fuite. Le pouvoir de cette matrone est d'autant plus efficace qu'elle agit en coulisses. $\mathrm{Au}$ regard du chroniqueur monastique, l'épouse du seigneur, maîtresse de l'intérieur du château, règne sur la sphère privée du lignage : les hommes la craignent d'autant plus qu'elle opère de manière insidieuse, secrète.

Le troisième personnage féminin à entrer sur scène est une veuve retrouvant son enfant perdu. La pilosité de Guifred dans un endroit exceptionnel, qu'elle est seule à savoir, lui permet une identification sûre du fils de ses entrailles : le motif folklorique de

17. G. M. Spiegel, aThe Reditus Regni ad Stirpem Karoli Magni. A New Look», French Historical Studies, 1971, p. 145-174; C. Beaune, .Les Capétiens et la naissance de l'histoire nationale», dans Le miracle capérien, S. Rials éd., Paris, 1987, p. 73. Sur la continuité entre Carolingiens et Capétiens, à travers la seule fonction royale, cf. B. Guenée, «Les généalogies entre l'histoire et la politique : la fierté d'être capétien, en France, au Moyen Âge», Annales. Économies. Sociétés. Civilisation, 1978, p. 450-477. 
la reconnaissance du personnage longtemps absent grâce à un trait corporel distinctif est à l'origine du surnom dont on pare l'ancêtre fondateur ${ }^{18}$. Mais son hirsutisme ne répondrait-il pas également à la volonté de l'auteur des Gesta d'exalter l'origine royale de la race du comte de Barcelone? Chacun connaît les cheveux longs des rois barbares, attribut de leur fonction de commandement. Dans un texte célèbre, Anastase le Bibliothécaire (800-879) décrit la crinière des Mérovingiens, à qui a les poils poussaient le long de l'épine dorsale comme des porcs ${ }^{19}$. En outre, cette pilosité reflétait, d'une bien voyante façon, l'antagonisme, que le chroniqueur tentait d'imposer dans les esprits, entre le comte Guifred ale Velu s et le maître dont il rejetait la domination, chez qui l'on reconnaissait aisément le roi Charles « le Chauve $~^{20}$. Quoi qu'il en soit, une fois identifié par sa mère, Guifred est présenté, en secret, aux magnats et aux barons de la principauté qui le reconnaissent pour leur seigneur. L'intervention de l'ancienne comtesse lui ouvre l'accès au pouvoir.

Cette forte femme conserve le privilège de convoquer l'assemblée des nobles de ses terres et de leur imposer son fils, malgré la présence du comte intrus : la viduité lui accorde une large autorité. Pour ce qui est du statut de la femme, notre texte traduit un imaginaire historiographique en plein accord avec les pratiques sociales du temps. La condition de la princesse, au cours de ses trois âges, y est nettement exprimée : passivité de la jeune fille nubile, pouvoir domestique de la mariée et puissance publique de la veuve.

Dans ce qu'il nous apprend du mariage entre Guifred I ${ }^{\text {er }}$ et Guinedilda, la fiction s'écarte, en revanche, du fait historique concret : au IX' siècle, leur union, comme presque toutes celles de l'aristocratie catalane de la période, avait été endogamique, isogamique et proche; dans la légende du XII siècle, elle est devenue exogamique (nul lien de parenté n'existe avec la maison de Flandre), hypergamique (le jeune guerrier épouse la fille du seigneur qui l'a élevé) et éloignée (les conjoints proviennent de la plus méridionale et de la plus septentrionale des provinces de la Francie occidentale). Ce décalage entre la représentation du mariage et sa réalité quotidienne disparaît, néanmoins, si nous nous plaçons à l'époque de la rédaction des Gesta : l'interdit de l'inceste était alors

18. M. Coll, op. cit. (n. 8), p. 131.

19. Dicebantur sane ex genere illo descendentes cristatce, quod interpretatur trichorachati: pilos enim habebant natos in spina veluti porci , interpolation d'Anastase dans sa traduction de Théophane, Chronographia, C. de Boor éd., Leipzig, 1885, p. 272 et 402.

20. J. Calmette, «Notes sur Wilfred le Velu», Revista de archivos, bibliotecas y museos, 1901, p. 444 . 
mieux respecté, les chevaliers se mariaient souvent à des filles d'un rang supérieur au leur et le voyage du cortège nuptial devenait de plus en plus long. La légende de Guifred le Velu en dit, une fois encore, davantage sur le XII siècle où elle apparaît que sur le $\mathrm{IX}^{\mathrm{e}}$ siècle qu'elle est supposée décrire.

C'est sur le problème du libre consentement des mariés que le texte renferme la plus surprenante des discordances entre les représentations mentales et l'exercice de la parenté, introduisant une profonde césure entre l'imaginaire de l'alliance et les pratiques oppressantes qui déterminent, au jour le jour, la désignation du conjoint. C'est en pleine indépendance, faisant fi de leur contraignant entourage, que Guifred et la fille du comte de Flandre se sont choisis l'un l'autre. Ce passage des Gesta, favorable, malgré les apparences, au modèle ecclésiastique, va bien plus loin : il conteste ouvertement les stratégies matrimoniales élaborées par le père ou l'aîné et présente le mariage consensuel comme la plus honorable des solutions. Face aux contraintes de la solidarité lignagère, la fiction demeure encore le seul exutoire. En ce qui concerne l'alliance médiévale, le mariage entre l'idéal et le matériel est impossible.

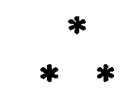

MM. Philippe Contamine, Robert-Henri BAUTIER, Jacques FonTAINE, Bernard GuEnÉE et Georges LE RIDER interviennent après cette communication. 


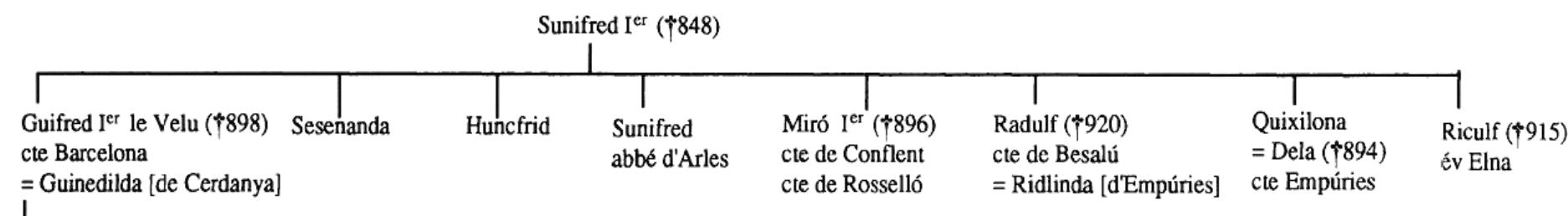

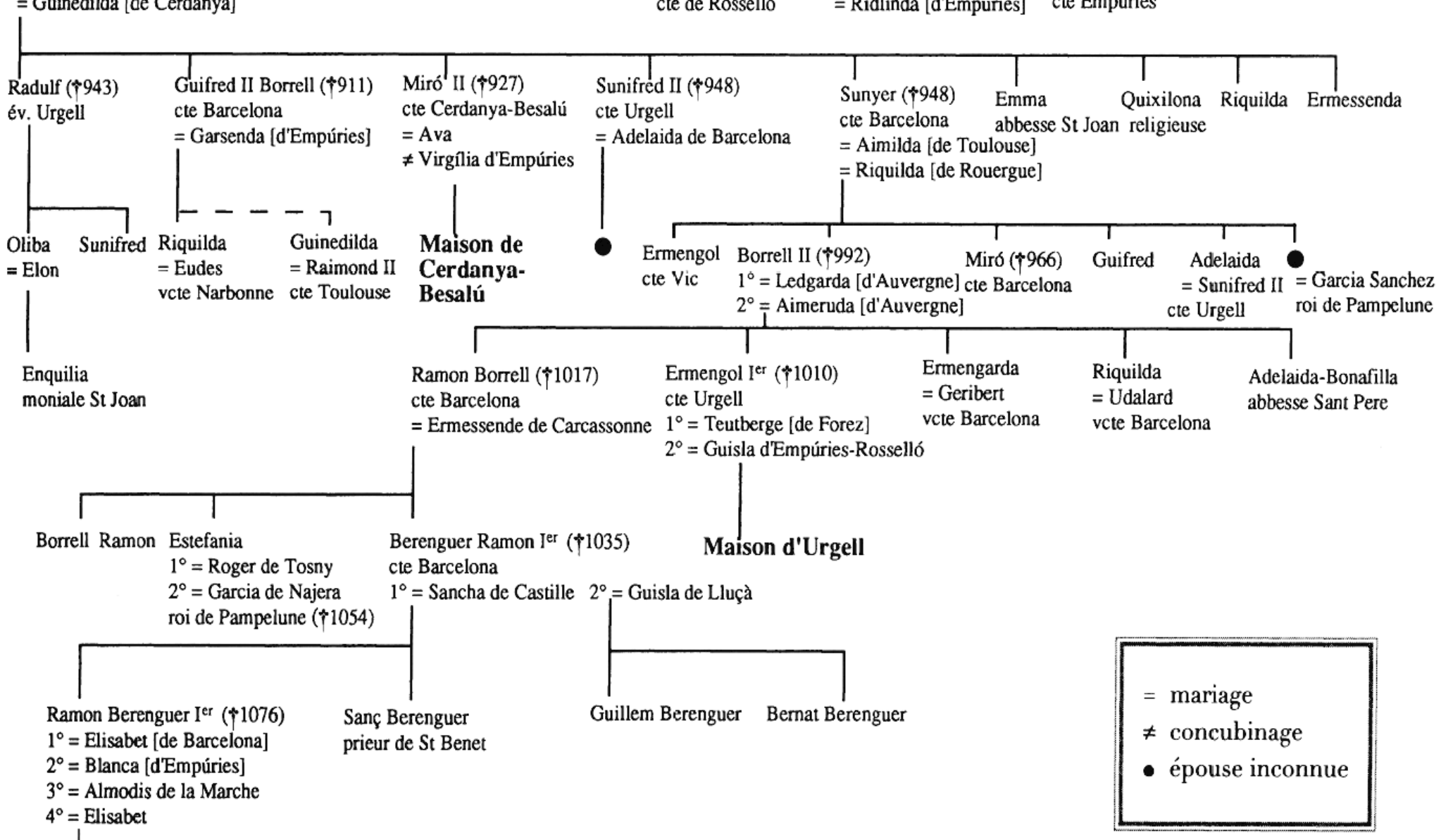

TABLEAU II.

Tabieau I. - Comtes de Barcelone ( $\mathbf{x}^{\mathrm{r}}-\mathrm{XI} \mathrm{I}^{\mathrm{e}}$ siècle). 


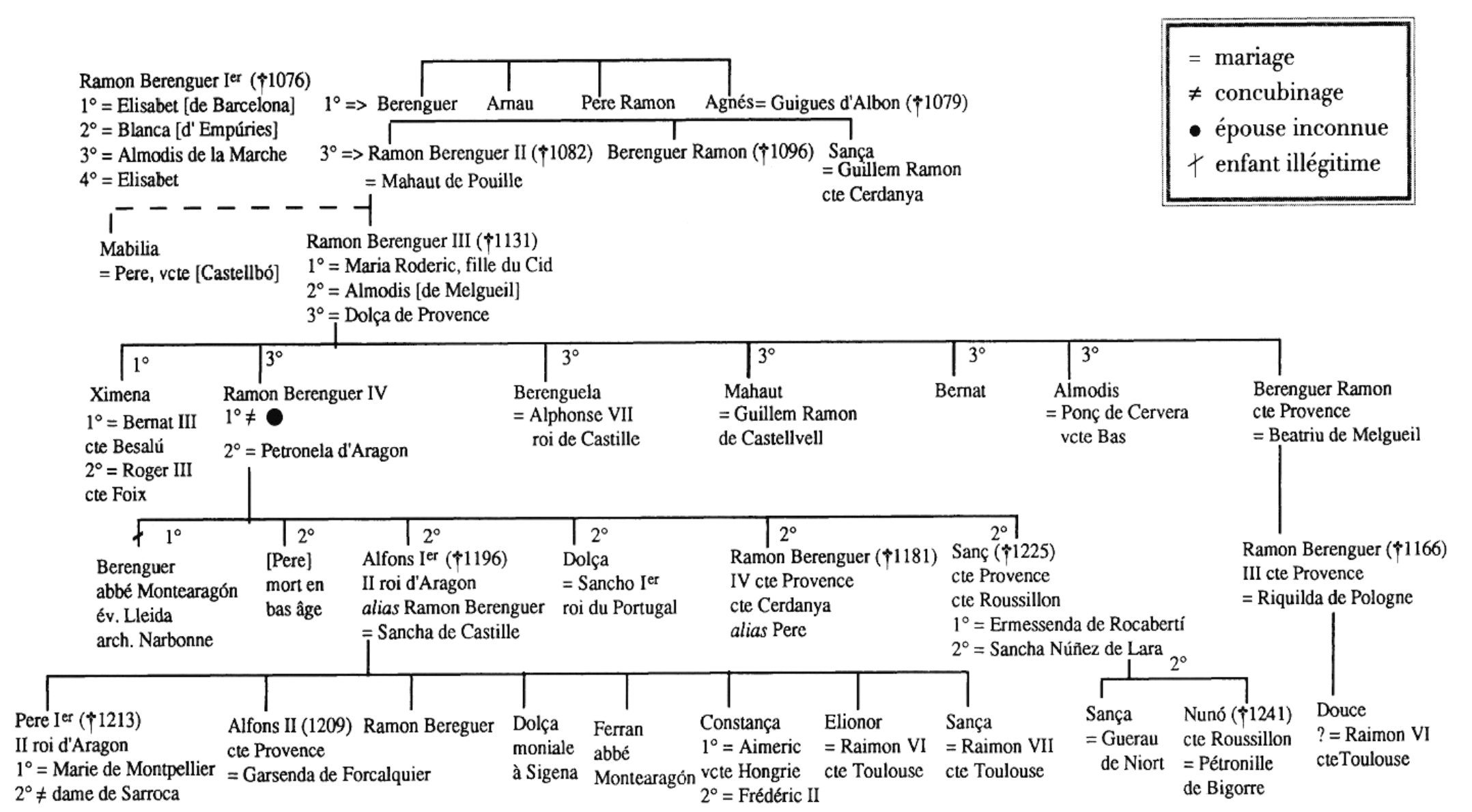

TABleal II. - Comtes de Barcelone (XIr siècle). 